\title{
The first-order deviation of superpolynomial in an arbitrary representation from the special polynomial
}

\author{
Anton Morozov1 \\ Moscow State University and ITEP, Moscow, Russia
}

\begin{abstract}
Like all other knot polynomials, the superpolynomials [1] should be defined in arbitrary representation $\mathrm{R}$ of the gauge group in (refined) Chern-Simons theory [2, 3. However, not a single example is yet known of a superpolynomial beyond symmetric or antisymmetric representations. Following [4, we consider the expansion of the superpolynomial around the special polynomial in powers of $q-1$ and $t-1$ and suggest a simple formula for the first-order deviation, which is presumably valid for arbitrary representation. This formula can serve as a crucial lacking test of various formulas for non-trivial superpolynomials, which will appear in the literature in the near future.
\end{abstract}

As it was shown in the article [5], some superpolynomials in the case of symmetrical and antisymmetrical representations possess simple factorization properties in the case of $q=1$ and $t=1$ respectively, which extend the corresponding property of the ordinary special polynomial [6, 7]. Now this property was also checked in [8] for all twisted knots, but there are arguments, that it does not hold for some more complicated knots [9].

There can be three directions to continue this research:

- to look at the other knots,

- to see what happens if $q$ or $t$ deviate from unity: $q=1+\hbar+\ldots$ or $t=1+\bar{\hbar}+\ldots$

- and to look at arbitrary representations.

The third direction is most interesting, but the problem is that we do not have any examples of superpolynomials even in the case of representation $[2,1]$ and as a result we can not really check our conjectures. Thus we choose a way in between: try to imagine, what the answer could be for the infinitesimal deviation from special polynomials, but for arbitrary representation and, perhaps, for generic knots.

Let us parameterize the small deviations of $q$ and $t$ from unity as follows:

$$
q=e^{\hbar} ; \quad t=e^{\bar{\hbar}}
$$

In this parametrization our superpolynomial can be written as $P_{R}(A, \hbar, \bar{\hbar})$. The special polynomial arises at $\hbar=0$ or $\bar{\hbar}=0$. and it satisfies [7]

$$
P_{R}(A, 0,0)=H_{R}(A, 0)=\left(H_{\square}(A, 0)\right)^{|R|}
$$

where $\square$ denotes the fundamental representation, and $|R|$ is the number of boxes in the Young Diagram of representation $R$.

In the next approximation we have:

$$
P_{R}(A, \hbar, \bar{\hbar})=\sigma_{\square}^{|R|}(A)+\hbar \eta_{R}(A)+\bar{\hbar} \bar{\eta}_{R}(A)+\ldots
$$

\footnotetext{
1anton.morozov@itep.ru
} 
where $\sigma_{\square}=H_{\square}(A, 0)$, and $\eta_{R}=\left.\frac{\partial\left(P_{R}(A, \hbar, 0)\right)}{\partial \hbar}\right|_{\hbar=0}, \bar{\eta}_{R}=\left.\frac{\partial\left(P_{R}(A, 0, \bar{\hbar})\right)}{\partial \bar{\hbar}}\right|_{\hbar=0}$.

Now let us see what can be said about the functions $\eta$ and $\bar{\eta}$. For symmetric representations we use the factrorization property [5] of the special superpolynomial (assuming that it is true for our knot):

$$
P_{[r]}(A, 0, \bar{\hbar})=\left(P_{\square}(A, 0, \bar{\hbar})\right)^{r}
$$

This relation is conjecturally true for all $\bar{\hbar}$, but we need it only in the first order, when it implies:

$$
\bar{\eta}_{[r]}(A)=r \sigma_{\square}^{r-1}(A) \bar{\eta}_{\square}(A)
$$

Similarly [5], for antisymmetric representation

$$
P_{\left[1^{r}\right]}(A, \hbar, 0)=\left(P_{\square}(A, \hbar, 0)\right)^{r}
$$

implies

$$
\eta_{1^{r}}(A)=r \sigma_{\square}^{r-1}(A) \eta_{\square}(A)
$$

Another piece of information comes from [4]. Namely, in the HOMFLY case, when $(\hbar=\bar{\hbar})$ we have

$$
\eta_{R}(A)+\bar{\eta}_{R}(A)=\varkappa_{R} \sigma_{\square}^{|R|-2} \sigma_{2}(A)
$$

where $\varkappa_{R}=\nu_{\bar{R}}-\nu_{R}$ and $\nu_{R}=\sum_{i} r_{i}(i-1)$. Here $r_{i}$ is a height of the column number $i$ in the Young diagram of the representation $R$. Finally, $\sigma_{2}(A)$ is the second special polynomial, like $\sigma_{\square}(A)$ it depends on the knot.

It is instructive to see how the reflection symmetry acts on the $\eta$-functions. According to [7] (see also [9] and references therein), it interchanges $R \leftrightarrow \bar{R}$ and also $q \leftrightarrow \frac{1}{t}$. Then $\hbar \leftrightarrow-\bar{\hbar}$ and the symmetry implies that

$$
\begin{aligned}
& \eta_{R} \rightarrow-\bar{\eta}_{\bar{R}} \\
& \bar{\eta}_{R} \rightarrow-\eta_{\bar{R}}
\end{aligned}
$$

Since for the fundamental representation $\square=\bar{\square}$, we see that $\overline{\eta_{\square}}=-\eta_{\square}$ and

$$
P_{\square}=\sigma_{\square}+\hbar \eta_{\square}+\bar{\hbar} \bar{\eta}_{\square}+\ldots=\sigma_{\square}+\hbar \eta_{\square}-\bar{\hbar} \eta_{\square}+\ldots=\sigma_{\square}-\hbar \bar{\eta}_{\square}+\bar{\hbar} \bar{\eta}_{\square}+\ldots
$$

Now we can summarize what we know about symmetric and antisymmetric representations. From (3) we have

$$
P_{[r]}=\sigma_{[r]}+\hbar \eta_{[r]}+\bar{\hbar} \bar{\eta}_{[r]}+\ldots
$$

while from (8) we know that

$$
\eta_{[r]}=\varkappa_{R} \sigma_{\square}^{|R|-2} \sigma_{2}-\bar{\eta}_{[r]}
$$


Substituting one into another, we get:

$$
P_{[r]}=\sigma_{\square}^{r}+\bar{\eta}_{[r]}(\bar{\hbar}-\hbar)+\hbar \sigma_{\square}^{r-2} \sigma_{2} \varkappa_{[r]}+\ldots=P_{\square}^{r}+\hbar \varkappa_{[r]} \sigma_{\square}^{r-2} \sigma_{2}+\ldots
$$

Similarly, for the antisymmetrical case:

$$
P_{\left[1^{r}\right]}=\sigma_{\square}^{r}+\eta_{\left[1^{r}\right]}(\hbar-\bar{\hbar})-\bar{\hbar} \varkappa_{\left[1^{r}\right]} \sigma_{\square}^{r-2} \sigma_{2}+\ldots=P_{\square}^{r}-\bar{\hbar} \varkappa_{\left[1^{r}\right]} \sigma_{\square}^{r-2} \sigma_{2}+\ldots
$$

Now, let us compare these two formulas. They differ: one contains $\hbar$ and another $\bar{\hbar}$, but now we can observe that for symmetric and antisymmetric representations $\varkappa_{R}$ is rather special: since $\nu_{[r]}=0, \varkappa_{[r]}=-\varkappa_{\left[1^{r}\right]}=\nu_{\left[1^{r}\right]}=\nu_{\overline{[r]}}$ and both formulas can be rewritten in a unified form:

$$
P_{R}=P_{\square}^{|R|}+\left(\hbar \nu_{\bar{R}}-\bar{\hbar} \nu_{R}\right) \sigma_{\square}^{|R|-2} \sigma_{2}+\ldots
$$

This is a remarkable formula, because in this form it can be used for arbitrary representation, not obligatory symmetric and antisymmetric. This formula is our new conjecture for the first deviation of arbitrary superpolynomial from the special one. At the moment there is no way to test this formula, because nothing is known yet about the superpolynomials beyond (anti)symmetric representations. For the first attempt to make use of (16) - in the case of the figure-eight knot and $R=[2,1]$, and with a somewhat controversial result - see [10]. Further advances in this direction are very desirable.

\section{Acknowledgements}

I am indebted to Alexei Morozov for useful discussions. My work is partly supported by the Ministry of Education and Science of the Russian Federation under the contract 8498, by the grants NSh-3349.2012.2, RFBR-12-01-00525.

\section{References}

[1] N.M.Dunfield, S.Gukov and J.Rasmussen, The Superpolynomial for Knot Homologies, Experimental Math. 15 (2006) 129159, math/0505662

[2] V.F.R.Jones, Index for subfactors, Invent.Math. 72 (1983) 1; A Polynomial Invariant for Knots via von Neumann Algebras, Bull.AMS 12 (1985) 103; Hecke Algebra Representations of Braid Groups and Link Polynomials, Ann.Math. 126 (1987) 335;

E.Witten, Quantum field theory and the Jones polynomial, Commun.Math.Phys. 121 (1989) 351;

M. Khovanov, A categorification of the Jones polynomial, Duke Math.J. 101 (2000) no.3, 359426, math/9908171

[3] R.Gopakumar and C.Vafa, On the Gauge Theory/Geometry Correspondence, Adv.Theor.Math.Phys. 3 (1999) 1415-1443, hep-th/9811131;

H.Ooguri and C.Vafa, Knot invariants and topological strings, Nucl.Phys. B577 (2000) 419-438, hep-th/9912123;

M.Aganagic, A.Klemm, M.Marino and C.Vafa, The Topological Vertex, Comm. Math. 
Phys. 254 (2005) 425-478, hep-th/0305132;

S.Gukov, A.Schwarz and C.Vafa, Khovanov-Rozansky Homology and Topological Strings, Lett. Math. Phys. 74 (2005) 5374, hep-th/0412243;

A.Iqbal, C.Kozcaz and C.Vafa, The Refined Topological Vertex, JHEP 0910:069, 2009, hep-th/0701156;

M.Aganagic, M.C.N.Cheng, R.Dijkgraaf, D.Krefl and C.Vafa, Quantum Geometry of Refined Topological Strings, arXiv:1105.0630;

M.Aganagic and Sh.Shakirov, Knot Homology from Refined Chern-Simons Theory, arXiv:1105.5117; Refined Chern-Simons Theory and Knot Homology, arXiv:1202.2489; Refined Chern-Simons Theory and Topological String, arXiv:1210.2733

[4] A.Morozov, A.Mironov and A.Sleptsov, to appear;

A. Mironov and A. Morozov, Equations on knot polynomials and 3d/5d duality, arXiv:1208.2282, sec.7.1

[5] Ant.Morozov, Supepolynomials and their representation dependence, arXiv:1208.3544

[6] K.Liu, P.Peng, Proof of the Labastida-Marino-Ooguri-Vafa Conjecture, arXiv:0704.1526; Sh.Zhu, Colored HOMFLY polynomial via skein theory, arXiv:1206.5886

[7] P.Dunin-Barkovsky, A.Mironov, A.Morozov, A.Sleptsov and A.Smirnov, Superpolynomials for torus knots from evolution induced by cut-and-join operators, arXiv:1106.4305

[8] S.Nawata, P.Ramadevi and Zodinmawia, Super-A-polynomials for Twist Knots, arXiv:1209.1409

[9] S.Gukov and M.Stosic, Homological algebra of knots and BPS states, arXiv:1112.0030

[10] A.Anokhina, A.Mironov, A.Morozov and And.Morozov, Knot polynomials in the first non-symmetric representation, arXiv:1211.6375 